\title{
DEGREE-ONE MAPS, SURGERY AND FOUR-MANIFOLDS
}

\author{
SIDDHARTHA GADGIL
}

\begin{abstract}
We give a description of degree-one maps between closed, oriented 3-manifolds in terms of surgery. Namely, we show that there is a degree-one map from a closed, oriented 3-manifold $M$ to a closed, oriented 3-manifold $N$ if and only if $M$ can be obtained from $N$ by surgery about a link in $N$ each of whose components is an unknot.

We use this to interpret the existence of degree-one maps between closed 3manifolds in terms of smooth 4-manifolds. More precisely, we show that there is a degree-one map from $M$ to $N$ if and only if there is a smooth embedding of $M$ in $W=(N \times I) \#_{n} \overline{\mathbb{C} P^{2}} \#_{m} \mathbb{C} P^{2}$, for some $m \geq 0, n \geq 0$ which separates the boundary components of $W$. This is motivated by the relation to topological field theories, in particular the invariants of Ozsvath and Szabo.
\end{abstract}

\section{IntRoduction}

We assume that all manifolds are connected and that all 3-manifolds are smooth. For closed, oriented 3-manifolds $M$ and $N$, we say that $M$ dominates $N$ (or $M$ 1dominates $N$ ) if there is a degree-one map from $M$ to $N$. This gives a transitive relation on closed, oriented 3-manifolds which has been extensively studied by several authors (for instance, see [1, [6], 12, 13], 14, 15], 18]). Note that every manifold dominates $S^{3}$ and that if $M$ dominates $N$ then there is a surjection from $\pi_{1}(M)$ to $\pi_{1}(N)$.

In this paper, we characterise dominance in terms of Dehn surgery. We use this to interpret dominance in terms of smooth 4-manifolds. The latter is motivated by the relation to topological field theories, in particular the invariants of Ozsvath and Szabo [10]11.

Date: October 25, 2018.

1991 Mathematics Subject Classification. Primary 57N10 ; Secondary 57N13, 57M27.

Key words and phrases. Degree-one maps, Dehn Surgery, Topological Field theories. 
Suppose $N$ is a closed 3-manifold and $M$ is obtained from $N$ by surgery about a link in $N$ all of whose components are homotopically trivial, then it is easy to see that there is a degree-one map from $M$ to $N$. Our first result is the converse, namely that if there is a degree-one map from $M$ to $N$, then $M$ can be obtained from $N$ by surgery about a link $L \subset N$ all of whose components are homotopically trivial. In fact we can find $L$ each of whose components is an unknot.

Theorem 1.1. For closed oriented 3-manifolds $M$ and $N$, there is a degree-one map from $M$ to $N$ if and only if $M$ can be obtained from $N$ by surgery about a link in $N$ each of whose components is an unknot in $N$.

We next interpret dominance of 3-manifolds in terms of 4-manifolds. Observe that a partial ordering on closed orientable 3-manifolds can be defined by saying that $M$ strongly dominates $N$ if there is a smooth embedding $i: M \rightarrow N \times(0,1) \subset$ $N \times[0,1]$ so that $i(M)$ separates the two boundary components $N \times\{0\}$ and $N \times\{1\}$, with the appropriate orientation. Observe that if $M$ strongly dominates $N$, the composition $\pi \circ i$ of the embedding $i$ with the projection $\pi: N \times[0,1] \rightarrow N$ has degree \pm 1 . We say that the embedding has the appropriate orientation if the degree of this map is one. Such a definition is related to the theory of imitations introduced by Kawauchi 8 .

This definition is motivated by the relation to $((3+1)$-dimensional $)$ topological field theories, in particular the invariants of Ozsvath and Szabo (however, our methods do not apply to the Ozsvath-Szabo theory because of the dependence on $\operatorname{Spin}^{c}$ structures). Recall that a degree-one map $f: M \rightarrow N$ induces a surjection $f_{*}$ on the level of fundamental groups. Hence if $\pi_{1}(N)$ is non-trivial so is $\pi_{1}(M)$. Further $f^{*}: H^{*}(N) \rightarrow H^{*}(M)$ is an injection, which shows that if $H^{k}(N) \neq 0$ then $H^{k}(M) \neq 0$.

We see that an analogous result holds for any topological field theory, with dominance replaced by strong dominance. Recall that a $(3+1)$-dimensional topological field theory associates to each closed, oriented 3-manifold $M$ a vector space $V(M)$ and to each cobordism $W$ from $M$ to another closed, oriented 3-manifold $N$ a linear 
transformation $T(W): V(M) \rightarrow V(N)$. Further this satisfies functorial properties, namely a product cobordism induces the identity map and if $W_{1}$ is a cobordism from $M_{1}$ to $M_{2}$ and $W_{2}$ is a cobordism from $M_{2}$ to $M_{3}$ then for the cobordism $W_{1} \coprod_{M_{2}} W_{2}$ from $M_{1}$ to $M_{3}, T\left(W_{1} \coprod_{M_{2}} W_{2}\right)=T\left(W_{2}\right) \circ T\left(W_{1}\right)$.

Suppose $M$ strongly dominates $N$, then splitting $N \times[0,1]$ along the given embedding of $M$ gives two cobordisms, $W_{1}$ from $N$ to $M$ and $W_{2}$ from $M$ to $N$. The composition of these is the product cobordism $N \times[0,1]$, which induces the identity map on $T(N)$. It follows that the identity map on $V(N)$ factors through $V(M)$, and in particular $V(N) \neq 0$ implies that $V(M) \neq 0$. This is the analogue of the corresponding results for $\pi_{1}$ and $H^{*}$ with respect to degree-one maps. Thus strong dominance plays the same role in the bordism category as dominance in the homotopy category.

We shall see (in Proposition 3.1) that the relation of strong dominance is stronger than dominance. We show, however, that dominance is equivalent to a relation obtained using 4-manifolds similar to the above one except that we allow 'positive and negative blow-ups'.

Theorem 1.2. For closed orientable 3-manifolds $M$ and $N$, there is a degree-one map from $M$ to $N$ if and only if there is a smooth embedding of $M$ in int $(W)$, $W=(N \times I) \#_{n} \overline{\mathbb{C} P^{2}} \#_{m} \mathbb{C} P^{2}$ for some $m>0, n>0$ which separates the boundary components of $W$, with the embedding having the appropriate orientation.

There is a relation in between dominance and strong dominance which is of interest. Namely, we say that $M$ negatively dominates $N$ if there is an embedding of $M$ into $W=(N \times I) \#_{n} \overline{\mathbb{C} P^{2}}$, for some $n \geq 0$, which separates the two boundary components. This is of interest because the Ozsvath-Szabo invariants (as also the Seiberg-Witten invariants) behave well under blowing up.

We shall see (in Proposition 3.1) that the Poincaré homology sphere does not even negatively dominate $S^{3}$. We shall study negative dominance elsewhere.

Acknowledgements. I thank the referee for many helpful comments. 


\section{Degree-One maps And Surgery}

In this section, we give a proof of Theorem 1.1. Suppose $M$ is obtained from $N$ by surgery about a link $L \subset N$ each of whose components $K_{i}$ is homotopically trivial. Then it is shown in [1) that there is a degree-one map from $M$ to $N$.

The converse is based on the following theorem of Haken [5] and Waldhausen [16] (see also [14).

Theorem (Haken-Waldhausen). Let $f: M \rightarrow N$ be a degree-one map and let $N=H_{1} \cup H_{2}$ be a Heegaard decomposition of $N$ with $H_{1}$ and $H_{2}$ handlebodies. Then $f$ is homotopic to a map $g$ such that $\left.g\right|_{g^{-1}\left(H_{1}\right)}: g^{-1}\left(H_{1}\right) \rightarrow H_{1}$ is a homeomorphism.

Such a map is called a 1-pinch. Thus if $M$ dominates $N$, there is a 1-pinch $g: M \rightarrow N$.

Proof of Theorem 1.1. Assume $M$ dominates $N$ and let $g, H_{1}$ and $H_{2}$ be as above. Consider a collection of properly embedded discs $D_{i}, 1 \leq i \leq n$, in $H_{2}$ such that on splitting $H_{2}$ along $D_{i}$, we get a 3 -ball $B$. We can assume that $g$ is transversal to $D_{i}$ for all $i, 1 \leq i \leq n$. Let $F_{i}=g^{-1}\left(D_{i}\right)$ and let $P=g^{-1}\left(H_{2}\right)$. Note that $\left.g\right|_{\partial H_{2}}$ is a homeomorphism and hence $F_{i}$ consists of a compact surface with a single boundary component and a (possibly empty) collection of closed surfaces. First, note that by performing a homotopy of $g$ we can assume that each $F_{i}$ is connected. This follows (as the induced map on $\pi_{1}$ is a surjection) by using standard techniques using binding ties as in Stallings' proof of the Knesser conjecture (see for example the proof of Knesser's conjecture in [7]). Hence $F_{i}, 1 \leq i \leq n$, is a compact surface with a single boundary component.

We first consider the special case when each $F_{i}$ is a disc.

Lemma 2.1. Suppose $F_{i}=g^{-1}\left(D_{i}\right)$ is a disc for each $i, 1 \leq i \leq n$. Then $P$ is obtained from the handlebody $\mathrm{H}_{2}$ by surgery about a link, each of whose components is an unknot. 
Proof. After a homotopy of $g$, we can assume that $F_{i}$ maps homeomorphically onto $D_{i}$ for $1 \leq i \leq n$. On splitting $P$ along the properly embedded discs $F_{i}, 1 \leq i \leq n$, we get a manifold $\hat{P}$ with boundary a 2 -sphere. By the theorem of Lickorish and Wallace [9] [17, this can be obtained from $B$ by surgery about a link $L$ in $B$, with each component of $L$ an unknot. Thus $P$ is obtained from $H_{2}$ by surgery about a link, each component of which is an unknot.

It follows that, in this special case, $M$ is obtained from $N$ by surgery about a link each component of which is an unknot. We now turn to the general case.

In the general case, we shall perform surgery on $M$ to obtain a manifold $M^{\prime}$ and a degree-one map $g^{\prime}: M^{\prime} \rightarrow N$ which is as in the special case. Hence $M^{\prime}$ is obtained from $N$ by surgery about a link, each component of which is an unknot. Further $M$ is obtained from $M^{\prime}$ by surgery, so we get a link in $N$ so that surgery about this link gives $M$. We shall show that each component of this link is homotopically trivial in $N$. From this, we deduce that we can obtain $M$ from $N$ by surgery about a link, each component of which is an unknot.

First, we construct $M^{\prime}$ and $g^{\prime}$.

Lemma 2.2. There is a framed link $L^{\prime} \subset P \subset M$ such that, if $M^{\prime}$ is the result of surgery of $M$ about $L^{\prime}$, there is a degree-one map $g^{\prime}: M^{\prime} \rightarrow N$, which coincides with $g$ outside a neighbourhood of $L^{\prime}$, so that $g^{\prime-1}\left(D_{i}\right)$ is a disc for each $i$. Furthermore, if $P^{\prime} \subset M^{\prime}$ is the result of surgery of $P$ about $L^{\prime}$, then $g^{\prime}\left(P^{\prime}\right) \subset H_{2}$.

Proof. For each $i, 1 \leq i \leq n$, consider a collection $L_{i}$ of disjoint, embedded simple closed curves on $F_{i}$ which do not separate $F_{i}$ and are maximal with respect to this property. Then $L^{\prime}=\cup_{i} L_{i}$ is a link in $M$. We consider a corresponding framed link (also denoted $L^{\prime}$ ), with the framing of a component of $L_{i}$ given by the normal to $F_{i}$. Let $M^{\prime}$ be the manifold obtained from $M$ by surgery about the framed link $L^{\prime}$.

We shall see that the map $g$ induces a degree-one map $g^{\prime}$ from $M^{\prime}$ to $N$ with $g^{\prime-1}\left(D_{i}\right)$ obtained from $F_{i}$ by compressing along the components of $L_{i}$. By the choice of $L_{i}$ it follows that $g^{-1}\left(D_{i}\right)$ is a disc for all $i, 1 \leq i \leq n$. 
Let the components of $L_{i}$ be $C_{j}^{i}$ and let $T_{j}^{i}$ denote a regular neighbourhood of $C_{j}^{i}$. On the complement of $\bigcup_{i, j} \operatorname{int}\left(T_{j}^{i}\right)$, we let $g^{\prime}=g$. After surgery, each $T_{j}^{i}$ is replaced by a solid torus $X=X_{j}^{i}$ with the same boundary as $T_{j}^{i}$. Now, $F_{i} \cap \partial X=F_{i} \cap \partial T_{j}^{i}$ is the union of two parallel curves $\mu_{1}$ and $\mu_{2}$ (on $\partial X$ ). Furthermore, by the choice of the surgery slope, $\mu_{1}$ and $\mu_{2}$ are meridians in $X$,i.e., they bound properly embedded $\operatorname{discs} E_{1}$ and $E_{2}$ in $X$. As $g\left(\mu_{i}\right) \subset D_{i}$ and $\partial E_{i}=\mu_{i}$, the map $g^{\prime}$ extends to $E_{i}$ with $g^{\prime}\left(E_{i}\right) \subset D_{i}$. Using transversality of $g$ to $D_{i}$, we see that we can extend $g^{\prime}$ to a regular neighbourhood $E_{i} \times[-1,1]$ of $E_{i}$ with $g^{\prime}\left(E_{i} \times[-1,1]-E_{i}\right) \cap D_{i}=\phi$. Finally, $X-\left(E_{1} \times(-1,1)\right)-\left(E_{2} \times(-1,1)\right)$ is the union of two balls $B_{1}$ and $B_{2}$, each of whose boundaries consists of two discs (one a component of $E_{1} \times\{-1,1\}$ and one a component of $\left.E_{2} \times\{-1,1\}\right)$ and an annulus in $X$ disjoint from $\mu_{1}$ and $\mu_{2}$. The function $g^{\prime}$ has been defined on $\partial B_{i}$ for $i=1,2$ and, by construction and using the fact that $T_{j}^{i}$ is disjoint from $F_{l}$ for $l \neq i$, the image of $g^{\prime}\left(\partial B_{k}\right)$ is contained in $B$ for $k=1,2$. Thus $g^{\prime}$ extends to a map on $X$ with $g^{\prime}\left(B_{k}\right) \subset B, k=1,2$. It follows that $\left(\left.g^{\prime}\right|_{X}\right)^{-1}\left(D_{i}\right)=E_{1} \cup E_{2}$. Making this construction for each $X_{j}^{i}$, we get a map $g^{\prime}$ as claimed.

Now, by applying Lemma 2.2 to $g^{\prime}$, we see that $M^{\prime}$ can be obtained from $N$ by surgery about a link $L_{0} \subset H_{2} \subset N$, each of whose components is homotopically trivial in $H_{2} \subset N$. Surgery of $H_{2}$ about $L_{0}$ gives the manifold (with boundary) $P^{\prime}$. We now perform surgeries about knots $\gamma_{j}^{i} \subset P^{\prime} \subset M^{\prime}$ so that the surgery about $\gamma_{j}^{i}$ cancels the surgery about $C_{j}^{i} \subset L^{\prime}$. Thus on performing such surgeries we obtain $M$. As $P^{\prime}$ is obtained from $H_{2}$ by surgery and the knots $\gamma_{j}^{i} \subset K^{\prime}$ can be perturbed to be disjoint from the locus of the surgery, they can be regarded as knots in $H_{2} \subset N$. Thus the union of the knots $\gamma_{j}^{i}$, with framing corresponding to the canceling surgeries, is a framed link $L_{1} \subset H_{2} \subset N$.

Thus $M$ is obtained from $N$ be surgery about the framed link $L=L_{0} \cup L_{1}$, with each component of $L_{0}$ homotopically trivial. We next show that the knots $\gamma_{j}^{i}$, regarded as curves in $H_{2} \subset N$, are homotopically trivial. 
Lemma 2.3. The knots $\gamma_{j}^{i}$, regarded as curves in $H_{2} \subset N$, are homotopically trivial.

Proof. Recall that $\gamma_{j}^{i}$ is the knot corresponding to the surgery canceling the surgery about $C_{j}^{i}$. Hence it is obtained by pushing off a meridian of $T_{j}^{i}$. Thus, $\gamma_{j}^{i}$ intersects $F_{i}$ transversally in two points, with opposite signs of intersection, and $\gamma_{j}^{i}$ is disjoint from $F_{k}$ for $k \neq i$.

Note that $\pi_{1}\left(H_{2}\right)$ is a free group with generators $\alpha_{i}, 1 \leq i \leq n$, corresponding to the discs $D_{i}$. Further, if $\gamma$ is a curve transversal to the discs $D_{i}, 1 \leq i \leq n$, then (up to conjugacy) the word represented by $\gamma$ is determined by the intersection points with the discs $D_{i}$. Namely, if the points of $\gamma \cap\left(\cup_{i} D_{i}\right)$, in cyclic order around $\gamma$, are contained in $D_{i_{1}}, \ldots D_{i_{k}}$ with signs of intersection $\epsilon_{j}= \pm 1$, then $\gamma=\alpha_{i_{1}}^{\epsilon_{1}} \ldots \alpha_{i_{1}}^{\epsilon_{k}}$ up to conjugacy.

As $\gamma_{j}^{i}$ intersects $F_{i}$ (hence $D_{i}$ ) transversally in two points, with opposite signs of intersection, and $\gamma_{j}^{i}$ is disjoint from $F_{k}$ (hence $D_{k}$ ) for $k \neq i$, it follows that $\gamma_{j}^{i}$ represents the trivial word in $\pi_{1}\left(H_{2}\right)$, and hence is homotopically trivial in $N$.

Thus, $M$ is obtained from $N$ by surgery about a link, each component of which is homotopically trivial. We shall deduce from this that we can choose the link so that each component is an unknot.

Lemma 2.4. Suppose $M$ is obtained from $N$ by surgery about a link $L$, each component of which is homotopically trivial. Then $M$ is obtained from $N$ by surgery about a link $L^{\prime}$, each component of which is an unknot.

Proof. As each component of $L$ is homotopically trivial, there is a sequence of crossing changes so that on performing these crossing changes we obtain a link all of whose components are unknots. Observe that each crossing change of a knot $\kappa$ is locally of a standard form. Namely, there is a ball $B \subset M$ which intersects $\kappa$ in a pair of $\operatorname{arcs} c_{1}$ and $c_{2}$, and the crossing change corresponds to a crossing of these $\operatorname{arcs}$ to give new $\operatorname{arcs} c_{1}^{\prime}$ and $c_{2}^{\prime}$ with the same endpoints as $c_{1}$ and $c_{2}$. 
Further, if $K_{i}$ is an unknot in $B$ unlinked from the $\operatorname{arcs} c_{i}$ with framing \pm 1 , then on performing the Kirby moves of sliding $c_{1}$ and $c_{2}$ over $K_{i}$, with opposite orientations, we get the knot obtained by crossing $c_{1}$ and $c_{2}$. In this manner we can obtain both positive and negative crossing changes.

Replacing $L$ by its union with unknots and performing the Kirby moves as above does not change the resulting manifold. Thus, we can replace $L$ by a framed link in $N$, each of whose components is an unknot, so that the result of surgery about the link is $M$.

This completes the proof of Theorem 1.1

\section{SURGERY AND 4-MANIFOLDS}

We now characterise dominance in terms of 4-manifolds.

Proof of Theorem 1.2. Suppose $M$ embeds in $W$ as in the hypothesis. Then $W-M$ has two components with closures $K_{1}$ and $K_{2}$ so that $\partial\left[K_{1}\right]=[M]-[N \times\{0\}]$. Hence $[M]$ is homologous to $[N \times\{0\}]$. Now by identifying all the points in each $\mathbb{C} P^{2}$ and $\overline{\mathbb{C} P^{2}}$ in $W=(M \times I) \#_{n} \overline{\mathbb{C} P^{2}} \#_{m} \mathbb{C} P^{2}$ to a single point, we get a blowdown map $\pi: W \rightarrow N \times[0,1]$. By composing with the projection, we get a map $p: W \rightarrow N$ with $p: N \times\{0\} \rightarrow N$ being the identity map. This restricts to a map $p: M \rightarrow N$. As $[M]$ is homologous to $[N \times\{0\}], p_{*}([M])=[N]$, i.e., $M$ has degree one.

Conversely, assume $M$ and $N$ are as in the hypothesis. By Theorem 1.1, $M$ can be obtained from $N$ by surgery about a framed link $L$, all of whose components are unknots in $N$. Hence $L$ can be obtained from an unlink $L_{0} \subset N$ by a sequence of (say $p$ ) crossings.

Let $K_{1}, \ldots K_{n}$ a collection of unknots in $N$, with $n \geq p$ to be specified later, so that $L_{0} \cup\left\{K_{1}, \ldots K_{n}\right\}$ forms an unlink. Let $W$ be obtained by attaching a 2-handle with framing \pm 1 (with signs to be chosen later) to $N \times[0,1 / 2]$ along each of $K_{0}$, $K_{1}, \ldots K_{n}$. Note that $W=(N \times[0,1]) \#_{k} \overline{\mathbb{C} P^{2}} \#_{l} \mathbb{C} P^{2}$ for some $k$ and $l$. 
We shall construct a different Kirby diagram for $W$. Corresponding to the $p$ crossings of $L_{0}$ required to make it isotopic to $L$ we can find disjoint balls $B_{i}$, $1 \leq i \leq p$, in which the crossing is made. By an isotopy, we can assume that for $1 \leq i \leq p, K_{i}$ is contained in $B_{i}$. Performing the Kirby moves corresponding to the crossing changes in each of these $B_{i}$, we get a Kirby diagram for $W$ with a sublink isotopic to $L$. Furthermore, by performing the Kirby move of sliding over the unknots $K_{p+1}, \ldots, K_{n}$ (with framing \pm 1 ) we can ensure this sublink is isotopic to $L$ as a framed link (as such a Kirby move changes the framing by \pm 1 without changing the link). Consider the corresponding Morse function for $W$ with the 2handles corresponding to components of $L$ attached first. The level set on attaching $L$ is the result of surgery about $L$. But this is $M$, and hence we get an embedding of $M$ separating the boundary components of $W$.

We next see that strong dominance is not the same as dominance. As is well known, any 3 -manifold dominates the 3 -sphere. However, we see that $S^{3}$ is not a minimal element with respect to strong dominance or even negative dominance. This result has also been observed by Ding [2].

Proposition 3.1. For $n \geq 0$, there is no embedding of the Poincare homology sphere in $\left(S^{3} \times I\right) \#_{n} \overline{\mathbb{C} P^{2}}$ which separates the boundary components.

Proof. Note that the Poincaré homology sphere can be obtained from $S^{3}$ by surgery on the $E_{8}$ link, and hence, with one of its orientations, bounds a 4-manifold $W$ with positive definite intersection pairing. Denote the Poincaré homology sphere with this orientation as $M$.

Suppose, for some $n \geq 0$, there is an embedding of the Poincare homology sphere in $\left(S^{3} \times I\right) \#_{n} \overline{\mathbb{C} P^{2}}$ which separates the boundary components. Then by capping off the boundary components $S^{3} \times\{0\}$ and $S^{3} \times\{1\}$, we get an embedding of the Poincare homology sphere in $\#_{n} \overline{\mathbb{C} P^{2}}$. Splitting along the embedding and using the Mayer-Vietoris sequence, we get 4-manifolds $W_{1}$ and $W_{2}$ bounding $M$ and $-M$ with positive definite intersection forms. If $\partial W_{1}=-M$, then $Y=W \coprod_{M} W_{1}$ is 
a smooth 4-manifold with $H_{1}(Y, \mathbb{Z})=0$ and the intersection form on $H_{2}(Y, \mathbb{Z})$ is positive definite but not diagonalisable, contradicting Donaldson's theorem [3].

\section{REFERENCES}

1. M. Boileau and S. Wang, 'Non-zero degree maps and surface bundles over $S^{1}$, J. Differential Geom. 43 (1996), 789-806.

2. F. Ding, 'Smooth structures on some open 4-manifolds', Topology 36 (1997), no. 1, 203-207.

3. S. K. Donaldson, 'An application of gauge theory to four-dimensional topology', J. Differential Geom. 18 (1983), no. 2, 279-315.

4. M. Freedman, 'The topology of four-dimensional manifolds', J. Differential Geom. 17 (1982), $357-453$.

5. W. Haken, 'On homotopy 3-spheres', Illinois J. Math. 10 (1966) 159-178.

6. C. Hayat-Legrand, S. Wang and H. Zieschang, 'Any 3-manifold 1-dominates at most finitely many 3-manifolds of $S^{3}$-geometry', Proc. Amer. Math. Soc. 130 (2002), 3117-3123.

7. J. Hempel, '3-Manifolds', Ann. of Math. Studies, No. 86, Princeton University Press, Princeton, 1976.

8. A. Kawauchi, 'An imitation theory of manifolds', Osaka J. Math. 26 (1989), no. 3, 447-464.

9. W. B. R. Lickorish, 'A representation of orientable combinatorial 3-manifolds', Ann. of Math. (2) 76 (1962) 531-540.

10. P. Ozsvath, Peter and Z. Szabo, 'Holomorphic discs and topological invariants for closed three-manifolds', to appear in the Annals of Mathematics.

11. P. Ozsvath and Z. Szabo, 'Holomorphic triangles and invariants of smooth four-manifolds', preprint

12. A. W. Reid, and S. Wang 'Non-Haken 3-manifolds are not large with respect to mappings of non-zero degree', Comm. Anal. Geom. 7 (1999), 105-132.

13. Y. W. Rong, 'Degree one maps between geometric 3-manifolds', Trans. Amer. Math. Soc. 332 (1992), 411-436.

14. Y. W. Rong and S. Wang, 'The preimages of submanifolds', Math. Proc. Cambridge Philos. Soc. 112 (1992), 271-279.

15. T. Soma, 'Non-zero degree maps to hyperbolic 3-manifolds', J. Differential Geom. 49 (1998), $517-546$.

16. F. Waldhausen, 'On mappings of handlebodies and of Heegaard splittings', Topology of Manifolds (Proc. Inst., Univ. of Georgia, Athens, Ga., 1969) 205-211 
17. A. D. Wallace, 'Modifications of cobounding manifolds', Canad. J. Math. 12, 503-528 (1960).

18. Wang, Shicheng; Zhou, Qing 'Any 3-manifold 1-dominates at most finitely many geometric 3-manifolds.' Math. Ann. 322 (2002), 525-535.

Department of Mathematics, Indian Institute of Science, Bangalore 560003, India

E-mail address: gadgil@math.iisc.ernet.in 